\title{
Betriebsräte, Tarifverträge und freiwillige Kündigungen von Arbeitnehmern
}

Die anhaltende Debatte über eine Reform des Kündigungsschutzgesetzes sowie über die Beschäftigungswirkungen von Mitbestimmung und Tarifverträgen hat zu einer Vielzahl von Studien geführt, die sich mit arbeitgeberseitigen Personalfreisetzungen befassen. Dabei gerät jedoch ein wichtiger Teil der Personalfluktuation aus dem Blickfeld - freiwillige Kündigungen durch die Arbeitnehmer selbst. Aber gerade dieser Aspekt liefert Anhaltspunkte, inwieweit das System industrieller Beziehungen in Deutschland die Arbeitszufriedenheit erhöht und somit zu einer Reduzierung arbeitnehmerseitiger Kündigungen beiträgt, was sich positiv auf die betriebliche Produktivität auswirken kann. Hierzu erfolgt im vorliegenden Beitrag neben einer theoretischen Betrachtung auch eine empirische Analyse.

\section{Einleitung}

Freiwillige Kündigungen von Arbeitnehmern machen einen erheblichen Teil der Personalfluktuation in deutschen Firmen aus. So haben nach Angaben des IAB-Betriebspanels für Westdeutschland etwa 348.000 Arbeitnehmer im ersten Halbjahr 2004 freiwillig gekündigt. Das sind fast $28 \%$ aller Personalabgänge (TNS Infratest 2005). Arbeitnehmerseitige Kündigungen sind oft mit Kosten für die Firmen verbunden, aber es besteht auch die Möglichkeit, dass freiwillige Kündigungen in Phasen des Personalabbaus erfolgen und von der Arbeitgeberseite sogar erwünscht sind und gefördert werden. Wenn jedoch die Arbeitsplätze neu besetzt werden, müssen neue Arbeitnehmer eingestellt und eingearbeitet werden, wodurch der Firma beispielsweise Suchkosten, administrative Kosten und Trainingskosten entstehen. Arbeitnehmerseitige Personalabgänge können außerdem mit dem Verlust von implizitem Wissen und betriebsspezifischem Humankapital einhergehen, in das die Firma investiert hat. Daher haben Firmen häufig ein Interesse daran, dass Arbeitnehmer nicht von sich aus kündigen.

Ebenso besteht von Seiten der Arbeitnehmer ein Interesse an stabilen Beschäftigungsverhältnissen, da ein Firmenwechsel auch für sie mit materiellen oder auch immateriellen Kosten verbunden ist. Als Beispiel für Letzteres ist der Verlust von sozialen Beziehungen am alten Arbeitsplatz zu nennen. Aufgrund des freiwilligen Charakters der arbeitnehmerseitigen Kündigung ist jedoch davon auszugehen, dass die
Arbeitnehmer einem gewissen ökonomischen Kalkül folgen und somit für ihre Kosten kompensiert werden. Mit anderen Worten: Ein Arbeitnehmer wird nur dann kündigen, wenn sein Nutzen aus einer Alternative größer ist als der Nutzen bei seinem derzeitigen Arbeitgeber. Eine mögliche Alternative kann der Wechsel zu einem anderen Arbeitgeber sein, bei dem die Arbeitsbedingungen besser sind (z. B. höherer Lohn, geringere Arbeitszeit, besseres Arbeitsklima, Gesundheitsschutz). Eine andere Alternative besteht im zeitweiligen oder dauerhaften Ausstieg aus dem Erwerbsleben, falls der Disnutzen aus dem Arbeitsleid oder der Nutzen aus Freizeit sehr hoch bewertet werden. Ein weiterer möglicher Kündigungsgrund sind private Motive, wie beispielsweise der Umzug der Familie in eine andere Region oder familiäre Verpflichtungen, wie Kindererziehung und Pflege von Familienangehörigen.

Während bei privaten Gründen die Einflussmöglichkeiten des Arbeitgebers zur Vermeidung einer freiwilligen Kündigung eher gering sind, kann er bei ökonomisch motivierten Kündigungen durch Verbesserung der Arbeitsbedingungen einen Wechsel der Arbeitnehmer in eine andere Firma oder in Arbeitslosigkeit abwenden. Allerdings muss die Firma über die Absichten und Präferenzen der Arbeitnehmer informiert sein, um eingreifen zu können. Bei der Informationsbeschaffung und Informationsbereitstellung kommt der kollektiven Arbeitnehmervertretung in Form von Betriebsräten eine zentrale Rolle zu. Ferner sind viele Arbeitsbedingungen, insbesondere Lohn und Arbeitszeit, in Tarifverträgen geregelt, sodass auch diese kollektiven Vereinbarungen Einfluss auf Kündigungs- entscheidungen haben sollten. Inwieweit die beiden Institutionen Betriebsrat und Tarifvertrag das Kündigungsverhalten von Arbeitnehmern beeinflussen, ist die zentrale Frage dieses Beitrages.

Der Beitrag gliedert sich wie folgt: Zunächst erfolgen einige theoretische Ausführungen zu den Ansätzen „Exit-Voice“ und "Collective Voice" sowie zu den Einflussmöglichkeiten von Betriebsräten und Gewerkschaften auf das arbeitnehmerseitige Kündigungsverhalten. In Abschnitt 3 wird der Stand der empirischen Forschung skizziert. Eine empirische Analyse mit Daten aus dem Hannoveraner Firmenpanel schließt sich in Abschnitt 4 an. Nach der Beschreibung des Datensatzes und der Methodik werden die Verbreitung von Tarifverträgen und Betriebsräten sowie die Ergebnisse der ökonometrischen Auswertungen zu den betrieblichen Determinanten freiwilliger Kündigungen dargestellt. Den Abschluss bildet ein kurzes Fazit.

Christian Pfeifer, Dipl.-Ök., wissenschaft-
licher Mitarbeiter an der Leibniz Universität
Hannover, Institut für Arbeitsökonomik.
Arbeitsschwerpunkte: Mikroökonomische
Arbeitsmarktanalysen, Personalökonomik,
empirische Arbeitsmarkt- und Personal-
forschung.
e-mail: pfeifer@aoek.uni-hannover.de.

Für hilfreiche Anregungen danke ich Knut Gerlach, Uwe Jiriahn sowie den Teilnehmern der GIRA-Jahrestagung 2006. 


\section{Theoretische Überlegungen}

\subsection{GRUNDGEDANKE "EXIT-VOICE“ UND „COLLECTIVE VOICE“}

Im Mittelpunkt der theoretischen Überlegungen steht die von Hirschman (1970) entwickelte Exit-Voice-Hypothese, die bereits in den 1970er Jahren zur Analyse von Gewerkschaftseffekten in den USA angewandt wurde (Freeman 1976; Addison/ Gerlach 1983; Freeman/Medoff 1984). ${ }^{1}$ Die Exit-Voice-Hypothese besagt, dass Arbeitnehmer bei Unzufriedenheit mit ihren Arbeitsbedingungen zwei Möglichkeiten haben: Sie können freiwillig kündigen (Exit: Abgang) oder ihre Unzufriedenheit äußern (Voice: Widerspruch). Wird die Unzufriedenheit geäußert und vom Arbeitgeber aufgegriffen, kann dies zu einer Verbesserung der Arbeitsbedingungen führen, wodurch freiwillige Kündigungen überflüssig werden. Da Verbesserungen der Arbeitsbedingungen häufig allen Arbeitnehmern zugute kommen können, haben sie teilweise den Charakter eines öffentlichen Gutes. Zudem können bei Ausübung der Voice-Option individuelle Kosten durch Kontaktaufnahme mit Vorgesetzten und in noch stärkerem Ausmaß durch Repressalien bei unliebsamen Äußerungen entstehen. Es kann daher zu einem Trittbrettfahrerproblem kommen, in dem es für einzelne Individuen rational ist, nicht die Voice-Option, sondern die ExitOption zu wählen. Das Trittbrettfahrerproblem kann jedoch durch Einführung einer kollektiven Interessenvertretung (Collective Voice: kollektives Sprachrohr) verringert werden. Einzelne Arbeitnehmer können ihre Unzufriedenheit gegenüber ihren Interessenvertretern äußern, die diese dann an das Management weiterleiten. Somit ist der einzelne Arbeitnehmer versteckt und geschützt und hat ferner nicht die Kosten der Kontaktaufnahme mit Vorgesetzten zu tragen. Außerdem können Interessen der Arbeitnehmer besser organisiert werden, und die Verhandlungsposition gegenüber dem Arbeitgeber ist im Kollektiv stärker.

\subsection{EINFLUSSMÖGLICHKEITEN VON BETRIEBSRÄTEN UND GEWERKSCHAFTEN}

Die Collective-Voice-Funktion wird in vielen Betrieben durch den Betriebsrat wahrgenommen, der in Betrieben mit mindestens fünf Beschäftigten gewählt werden kann, falls dies von der Belegschaft gewünscht wird (Müller-Jentsch 1995; Addison et al. 2004). Nach dem Betriebsverfassungsgesetz hat der Betriebsrat in vielen Bereichen nicht nur ein Informationsrecht, sondern auch ein Mitbestimmungsrecht. Beispielhaft seien hier nur die Festlegung von Betriebszeiten (Arbeitsanfang, Arbeitsende, Pausen, Betriebsferien) sowie die Beteiligung an Weiterbildungs-, Einstellungsund Entlassungsentscheidungen genannt. Die betriebliche Interessenvertretung erfolgt zwar kollektiv und kann nicht immer die individuellen Präferenzen jedes einzelnen Beschäftigten berücksichtigen, aber es gibt gute Gründe, nur von begrenzt heterogenen Präferenzen innerhalb der Belegschaft einer Firma auszugehen. Denn es existiert zum einen eine gewisse Selbstselektion der Arbeitnehmer in bestimmte Betriebe und zum anderen eine gemeinsame Sozialisation innerhalb eines Betriebes. Neben einer generellen Erhöhung des Nutzens der Arbeitnehmer durch bessere Arbeitsbedingungen kann der Betriebsrat auch einzelnen Arbeitnehmern behilflich sein, wenn diese unzufrieden sind und überlegen, den Betrieb zu verlassen. So kann der Betriebsrat bei der Kontaktaufnahme mit entsprechenden Vorgesetzten behilflich sein, den Arbeitnehmer bei einem Gespräch mit Vorgesetzten unterstützen oder sogar an Stelle des Arbeitnehmers Verhandlungen mit Vorgesetzten führen. Daraus folgt:

\section{- Hypothese 1: Betriebsräte reduzieren die Anzahl freiwilliger Kündigungen.}

Der Einfluss von deutschen Gewerkschaften, im Sinne von „Collective Voice“ $\mathrm{zu}$ handeln, ist begrenzt, da sie in der Regel über Tarifverträge nur Einfluss auf die Festsetzung von Rahmenrichtlinien in ganzen Branchen und Regionen haben. Zudem vertreten Gewerkschaftsfunktionäre teilweise auch gesamtwirtschaftliche und politische Interessen. Die Interessen von einzelnen Arbeitnehmern oder auch der Belegschaft eines einzelnen Betriebes werden dagegen nur bedingt vertreten. Somit ist auch fraglich, ob Gewerkschaften bzw. Tarifverträge einen Einfluss gemäß der ExitVoice-Hypothese haben. Allerdings können bessere Arbeitsbedingungen im Geltungsbereich eines Tarifvertrages dazu führen, dass die Wahrscheinlichkeit eines freiwilligen Ausstieges aus dem Erwerbsleben abnimmt, da der Nutzen aus Beschäftigung steigt (z. B. höherer Lohn und geringere Arbeitszeit). Das führt zur

- Hypothese 2: Eine Tarifbindung reduziert die Anzahl freiwilliger Kündigungen. Jedoch ist der Einfluss auf die individuelle Kündigungswahrscheinlichkeit der Arbeitnehmer geringer als der von Betriebsräten.

Tarifverträge haben den Vorteil, dass Verteilungskonflikte großteils außerhalb des Betriebes ausgefochten werden (Freeman/ Lazear 1995). Daher sollte in tarifgebundenen Firmen die Kooperation zwischen Betriebsrat und Unternehmensleitung besser sein als in Firmen, in denen aufgrund von fehlenden exogenen Rahmenrichtlinien innerbetriebliche Verteilungskämpfe stattfinden. Diese Überlegung wird durch empirische Analysen von Hübler/Jirjahn (2003) gestützt. Die beiden Autoren finden in getrennten Schätzungen für Betriebe mit und ohne Tarifbindung, dass Betriebsräte in tarifgebundenen Betrieben einen kleineren Einfluss auf die Lohnkosten und einen stärkeren Einfluss auf die Produktivität haben als in nicht tarifgebundenen Betrieben. In nicht tarifgebundenen Betrieben ist der Produktivitätseffekt des Betriebsrates sogar insignifikant. Hübler/Jirjahn (2003) führen dies darauf zurück, dass Betriebsräte sich in tarifgebundenen Betrieben stärker mit produktivitätssteigernden Aktivitäten befassen und weniger danach streben, sich ökonomische Renten anzueignen.

Trotz der formalen Unabhängigkeit von betrieblicher und gewerkschaftlicher Interessenvertretung unterstützen Gewerkschaften die Betriebsratsarbeit finanziell, beratend und personell (Müller-Jentsch

\footnotetext{
Es ist anzumerken, dass Gewerkschaften im amerikanischen Arbeitsmarkt betrieblich organisiert sind und somit gegenüber Deutschland eine Art Mischfunktion wahrnehmen. Denn zum einen verhandeln sie ebenso wie deutsche Gewerkschaften über Lohn und Arbeitszeit. Zum anderen nehmen sie die Funktion der betrieblichen Interessenvertretung wahr, die in Deutschland von Betriebsräten übernommen wird.
} 
1995, S. 61ff.). Dies kommt auch darin zum Ausdruck, dass viele Betriebsratsmitglieder nicht nur Gewerkschaftsmitglieder sind, sondern auch von den Gewerkschaften für die Betriebsratswahlen nominiert werden. Eine höhere Kooperationsbereitschaft von Seiten der Unternehmensleitung sowie eine weiterreichende Unterstützung durch Gewerkschaften können dann auch zu einer effektiveren Umsetzung der VoiceFunktion von Betriebsräten in tarifgebundenen Firmen führen. Daraus folgt

- Hypothese 3: Betriebsräte sind effektiver in der Vermeidung freiwilliger Kündigungen, falls auch eine Tarifbindung vorliegt.

\section{Stand der empirischen Forschung}

Eine erste empirische Untersuchung zum Einfluss von Gewerkschaften auf das freiwillige Kündigungsverhalten von Arbeitnehmern stammt von Freeman (1980) für die USA. Er findet in seiner Analyse von Personendaten, dass Gewerkschaftsmitglieder mit einer geringeren Wahrscheinlichkeit freiwillig kündigen und längere Betriebszugehörigkeiten haben als nicht gewerkschaftlich organisierte Arbeitnehmer, was für die Exit-Voice Hypothese spricht. Dieser Zusammenhang wird auch von Miller/Mulvey (1991) für Australien bestätigt, die ebenfalls Personendaten auswerten. Eine neuere Untersuchung von Addison/Belfield (2004) zeigt für Großbritannien, dass in Betrieben mit Gewerkschaften die Kündigungsraten um $34 \%$ geringer sind und die Dauer der Betriebszugehörigkeiten um 16 \% höher ist als in Betrieben ohne Gewerkschaften.

Für Deutschland ist, wie bereits in den theoretischen Ausführungen dargelegt, zwischen Betriebsräten und Tarifverträgen zu unterscheiden. Eine Voice-Funktion ist dabei eher der betrieblichen Interessenvertretung zuzurechnen, während Tarifverträge zu einer allgemeinen Verbesserung der Arbeitsbedingungen führen können. Kraft (1986) kommt in einer Untersuchung einer kleinen Stichprobe von Betrieben zu dem Ergebnis, dass individuelle Voice-Möglichkeiten negativ mit der Wahrscheinlichkeit einer hohen Rate freiwilliger Kündigungen korreliert sind, aber Betriebsräte und Ge- werkschaften darüber hinaus keinen signifikanten Einfluss haben. In einer weiteren Untersuchung mit Betriebsdaten findet Frick (1996) dagegen, dass die Existenz eines Betriebsrates die jährliche Rate der freiwilligen Kündigungen signifikant um 1,5 Prozentpunkte reduziert und der gewerkschaftliche Organisationsgrad keinen signifikanten Einfluss ausübt.

Die Ergebnisse von Frick (1996) werden allerdings von Kraft (2006) in einer Replikationsstudie infrage gestellt. Denn Kraft (2006) kommt mit dem von Frick (1996) genutzten Datensatz zu dem Ergebnis, dass die Existenz eines Betriebsrates keinen signifikanten Einfluss auf freiwillige Kündigungen hat. Dies führt Kraft (2006) auf die Abgrenzung der genutzten Stichprobe und methodische Probleme in den Schätzungen von Frick (1996) zurück. Beispielsweise zeigt Kraft (2006), dass die Ergebnisse sensitiv auf die Spezifikation der Variablen und die Art der Log-Transformation reagieren und dass zudem ein Tobit-Ansatz anstelle von klassisch linearen Regressionen genutzt werden sollte. Frick (2006) verteidigt seine Ergebnisse, indem er auf neuere empirische Studien verweist, die ebenfalls einen signifikant negativen Einfluss von Betriebsräten auf die Personalfluktuation finden (z. B. Dilger 2002; Frick/Möller 2003). Jedoch unterscheiden diese neueren empirischen Arbeiten nicht zwischen arbeitnehmerseitigen und arbeitgeberseitigen Kündigungen. Frick/Möller (2003) analysieren beispielsweise den Einfluss von Betriebsrat, Tarifbindung und deren Koexistenz auf die betriebliche Personalfluktuation. Sie kommen zu dem Ergebnis, dass die Interaktion von Betriebsrat und Tarifvertrag die Fluktuation am stärksten verringert. $^{2}$

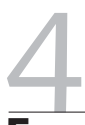

\section{Empirische Analyse}

\subsection{DATEN UND METHODIK}

Das Hannoveraner Firmenpanel ist seit dem Jahr 2000 die niedersächsische Teilstichprobe des IAB-Betriebspanels (Gerlach et al. 2003). In einer jährlichen Panelerhebung werden etwa 1.000 niedersächsische Betriebe mit mindestens einem sozialversicherungspflichtig Beschäftigten befragt. Die Stichprobe ist dabei nach Branchen und Betriebsgrößenklassen ge- schichtet und kann betriebsproportional hochgerechnet werden, sodass die deskriptiven Auswertungen repräsentativ sind für alle der rund 190.000 Betriebe in Niedersachsen, in denen fast 3 Mio. Arbeitnehmer beschäftigt sind. Für die ökonometrische Analyse wird ein ungewichtetes „unbalanced Panel“ für die Jahre 2000 bis 2004 verwendet. Gewichtete Regressionen sind nicht erforderlich, da Betriebsgröße und Branchen als Kontrollvariablen in den Schätzungen berücksichtigt werden. Der Vorteil des „unbalanced Panel“ ist, dass mehr Beobachtungen in den Schätzungen berücksichtigt werden und der Selektionsfehler für die Teilnahme an der Befragung verringert wird. Denn in einem „balanced Panel“würden nur Betriebe berücksichtigt, die in jedem Jahr des Beobachtungszeitraumes an der Befragung teilgenommen haben. Betriebe aus dem Non-Profit Sektor und öffentliche Verwaltungen werden aus der Analyse ausgeschlossen, da sie sich in den personalpolitischen Entscheidungen von privaten, gewinnorientierten Firmen unterscheiden und für sie einige Variablen nicht verfügbar sind. Neben Analysen für alle Betriebe werden getrennte Auswertungen für Betriebe in den Betriebsgrößenklassen 21 bis 100 Beschäftigte und 101 bis 500 Beschäftigte sowie für das Verarbeitende Gewerbe und die Dienstleistungsbranche vorgenommen.

In der ökonometrischen Analyse werden die betrieblichen Determinanten freiwilliger Kündigungen untersucht, wobei der Schwerpunkt auf dem Einfluss von Betriebsräten und Tarifverträgen liegt. Als abhängige Variable fungiert die logarithmierte Anzahl der freiwilligen arbeitnehmerseitigen Kündigungen $(Q)$ im ersten Halbjahr eines Jahres. ${ }^{3} \mathrm{Da}$ in recht vielen Betrieben keine arbeitnehmerseitigen Kündigungen vorkommen, würden diese Betriebe aus der Analyse ausgeschlossen werden, was zu einer Unterschätzung von Effekten zur Vermeidung von freiwilligen Kündigungen führen kann. Daher wird vor dem Logarithmieren zur Anzahl der Kündigungen

\footnotetext{
2 Ausführliche Überblicke zu theoretischen Begründungen und empirische Studien, die sich mit den ökonomischen Wirkungen von Betriebsräten befassen, sind in Addison et al. (2004), Jiriahn (2005) sowie Jirjahn (2006) zu finden.

3 Der Vorteil eines solchen exponentialen Funktionstyps ist, dass die Koeffizienten von Dummyvariablen $(\beta)$ leicht in prozentuale Änderungen umgerechnet werden können: $\left(e^{\beta}-1\right)^{*} 100$.
} 
Eins addiert $(Q+1)$. Zwar werden die Betriebe nun berücksichtigt, aber es besteht immer noch ein Problem linkszensierter Daten, sodass anstelle einer klassischen linearen Regression („Ordinary Least Squares") ein Tobit Modell mit einer Untergrenze von Null genutzt wird $(\ln (0+1)=0)$.

Aufgrund möglicher unbeobachtbarer Heterogenität zwischen den Betrieben wird neben einem gepoolten Tobit auch ein Random-Effects Tobit Modell geschätzt.

Als erklärende Variablen werden in einer ersten Spezifikation jeweils Dummyvariablen für die Existenz eines Betriebsrates und für eine Tarifbindung genutzt. Um jedoch auch einen Einblick zu gewinnen, inwieweit eine Koexistenz von Betriebsrat und Tarifvertrag freiwillige Kündigungen beeinflusst, werden in einer zweiten Spezifikation drei neue Variablen generiert. Diese geben an, ob der Betrieb nur einen Betriebsrat hat, nur tarifgebunden ist oder sowohl einen Betriebsrat hat als auch tarifgebunden ist. Die Referenzgruppe sind Betriebe, die weder einen Betriebsrat haben noch einem Tarifvertrag unterliegen.

Beide Spezifikationen beinhalten neben den Variablen zu den industriellen Beziehungen weitere Kontrollvariablen. ${ }^{4} \mathrm{Da}$ die Fluktuation in einem Betrieb von der wirtschaftlichen Situation abhängen kann, wird ein Indikator für betriebliche Nachfrageschocks aufgenommen. Dieser berechnet sich aus der Differenz des logarithmierten erwarteten Umsatzes des laufenden Geschäftsjahres und des logarithmierten tatsächlichen Umsatzes des abgelaufenen Geschäftsjahres. Ferner werden Dummyvariablen für erhebliche betriebliche Reorganisationen wie die Schließung, Ausgliederung oder Eingliederung eines Betriebsteils innerhalb des letzten Jahres berücksichtigt. Denn solche betrieblichen Änderungen haben auch Einfluss auf die Beschäftigungssituation von Arbeitnehmern. Unterschiede in der Personalstruktur der Betriebe werden durch die Anteile der Teilzeitbeschäftigten, weiblichen Beschäftigten, Arbeiter und Qualifizierten an allen Beschäftigten am 30. Juni eines jeden Jahres berücksichtigt. Aufgrund von Größenklassen- und Brancheneffekten werden die logarithmierte Anzahl der Beschäftigten am 30. Juni sowie deren quadratischer Term und elf Branchendummies aufgenommen. Um mögliche aggregierte Einflüsse in den einzelnen Jahren, wie die allgemeine konjunkturelle Lage, zu kontrollieren, werden zusätzlich Jahresdummies einbezogen.

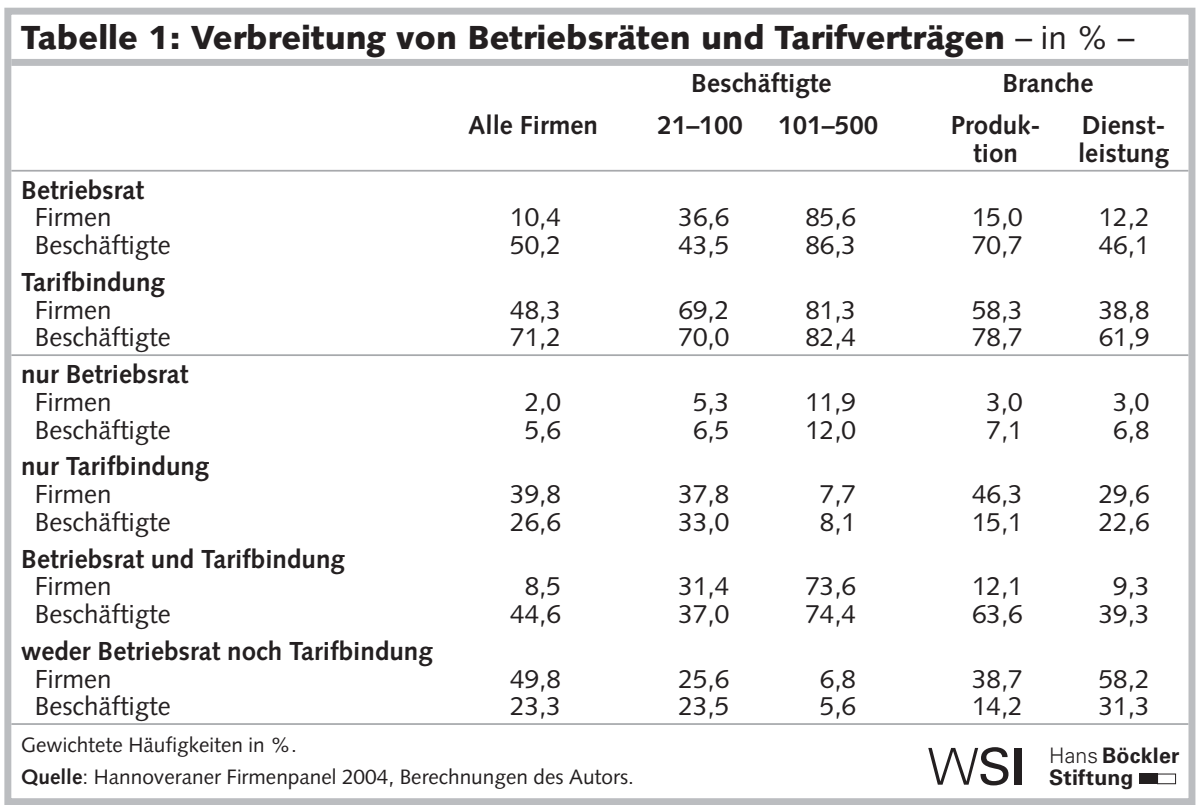

\subsection{VERBREITUNG VON BETRIEBS- RÄTEN UND TARIFVERTRÄGEN}

Tabelle 1 informiert zunächst über die Verbreitung von Betriebsräten und Tarifverträgen in Niedersachsen im Jahr 2004. Hierzu wurden die Daten aus dem Hannoveraner Firmenpanel hochgerechnet, um der Überrepräsentativität großer Betriebe in der Befragung Rechnung zu tragen. Etwa $10 \%$ aller Firmen, in denen aber die Hälfte aller Beschäftigten in Niedersachsen tätig ist, haben einen Betriebsrat. Der Größenklasseneffekt wird auch deutlich in den getrennten Auswertungen für zwei ausgewählte Größenklassen. Denn während fast $37 \%$ der Betriebe mit 21 bis 100 Beschäftigten einen Betriebsrat haben, sind es in Betrieben mit 101 bis 500 Beschäftigten schon fast $86 \%$. Ferner zeigt sich, dass aufgrund der Größenklasseneffekte der Anteil der Beschäftigten in Betrieben mit einem Betriebsrat im Verarbeitenden Gewerbe größer ist als in der Dienstleistungsbranche, in der die Betriebe tendenziell kleiner sind. Die Bindung an Tarifverträge ist deutlich stärker ausgeprägt. So sind über $48 \%$ aller Betriebe tarifgebunden. Erneut zeigt sich ein Größenklasseneffekt, da über $71 \%$ der Beschäftigten in tarifgebundenen Betrieben arbeiten. Dies ist auch in den Auswertungen nach Größenklassen und Branchen zu erkennen.

Der untere Teil von Tabelle 1 gibt einen genaueren Einblick in die Verbreitung von Betriebsräten und Tarifverträgen, indem zwischen vier Gruppen von Betrieben differenziert wird: nur Betriebrat, nur Tarifvertrag, sowohl Betriebsrat als auch Tarif- vertrag, weder Betriebsrat noch Tarifvertrag. Lediglich $2 \%$ aller Betriebe haben einen Betriebsrat und sind nicht an einen Tarifvertrag gebunden, wobei es sich tendenziell um größere Betriebe handelt. Der Anteil der Betriebe, die nur tarifgebunden sind, beträgt fast $40 \%$ und der Anteil der Beschäftigten in diesen Betrieben fast $27 \%$. Die 8,5 \% der Betriebe, die sowohl einen Betriebsrat haben als auch tarifgebunden sind, beschäftigen fast $45 \%$ aller Beschäftigten. Fast die Hälfte aller Betriebe hat weder einen Betriebsrat noch einen Tarifvertrag. Da hier aber nur $23 \%$ der Beschäftigten tätig sind, handelt es sich vor allem um kleinere Betriebe. Aber selbst in der Größenklasse mit 101 bis 500 Beschäftigten sind fast $7 \%$ der Betriebe nicht tarifgebunden und haben auch keinen Betriebsrat. Diese interessenvertretungsfreien Zonen sind insbesondere im Dienstleistungssektor weit verbreitet, wovon ungefähr $58 \%$ der Betriebe und $31 \%$ der Beschäftigten betroffen sind.

\subsection{ERGEBNISSE DER ÖKONO- METRISCHEN ANALYSE}

Tabelle 2 informiert über die Ergebnisse der einzelnen Tobit Schätzungen für die gesamte Stichprobe. Die Stichprobe umfasst

\footnotetext{
4 Aufgrund der Panelanalysen müssen die aufgenommenen Variablen in jeder Welle verfügbar sein, sodass möglicherweise einige weitere wichtige Variablen nicht berücksichtigt werden. Allerdings werden für solche Effekte teilweise im Random-Effects Modell kontrolliert.
} 
Tabelle 2: Betriebliche Determinanten freiwilliger Kündigungen

\begin{tabular}{|c|c|c|c|c|c|}
\hline & \multirow[b]{2}{*}{$\begin{array}{l}\text { Mittelwert } \\
\text { (pooled) }\end{array}$} & \multicolumn{2}{|c|}{ Spezifikation 1} & \multicolumn{2}{|c|}{ Spezifikation 2} \\
\hline & & $\begin{array}{c}\text { Tobit } \\
\text { (pooled) }\end{array}$ & $\begin{array}{c}\text { Tobit } \\
\text { (random } \\
\text { effects) }\end{array}$ & $\begin{array}{c}\text { Tobit } \\
\text { (pooled) }\end{array}$ & $\begin{array}{c}\text { Tobit } \\
\text { (random } \\
\text { effects) }\end{array}$ \\
\hline Betriebsrat (Dummy) & $\begin{array}{c}0,483 \\
{[0,500]}\end{array}$ & $\begin{array}{c}-0,326 * * * \\
{[0,071]}\end{array}$ & $\begin{array}{c}-0,280 * * * \\
{[0,077]}\end{array}$ & & \\
\hline Tarifbindung (Dummy) & $\begin{array}{c}0,684 \\
{[0,465]}\end{array}$ & $\begin{array}{c}-0,129 * * \\
{[0,064]}\end{array}$ & $\begin{array}{l}-0,101 \\
{[0,068]}\end{array}$ & & \\
\hline nur Betriebsrat (Dummy) & $\begin{array}{c}0,063 \\
{[0,243]}\end{array}$ & & & $\begin{array}{c}-0,237^{* *} \\
{[0,115]}\end{array}$ & $\begin{array}{c}-0,218^{*} \\
{[0,123]}\end{array}$ \\
\hline nur Tarifbindung (Dummy) & $\begin{array}{c}0,263 \\
{[0,441]}\end{array}$ & & & $\begin{array}{l}-0,079 \\
{[0,082]}\end{array}$ & $\begin{array}{l}-0,067 \\
{[0,085]}\end{array}$ \\
\hline $\begin{array}{l}\text { Betriebsrat und Tarifbindung } \\
\text { (Dummy) }\end{array}$ & $\begin{array}{c}0,420 \\
{[0,494]}\end{array}$ & & & $\begin{array}{c}-0,440 * * * \\
{[0,083]}\end{array}$ & $\begin{array}{c}-0,372 * * * \\
{[0,090]}\end{array}$ \\
\hline Nachfrageschock $\left(\log Y_{t}{ }_{t}-\log Y_{t-1}\right)$ & $\begin{array}{l}-0,059 \\
{[0,976]}\end{array}$ & $\begin{array}{l}-0,040 \\
{[0,026]}\end{array}$ & $\begin{array}{l}-0,035 \\
{[0,025]}\end{array}$ & $\begin{array}{l}-0,041 \\
{[0,026]}\end{array}$ & $\begin{array}{l}-0,036 \\
{[0,025]}\end{array}$ \\
\hline Betriebsteil geschlossen (Dummy) & $\begin{array}{c}0,029 \\
{[0,167]}\end{array}$ & $\begin{array}{c}0,374 * * * \\
{[0,135]}\end{array}$ & $\begin{array}{c}0,351 * * * \\
{[0,128]}\end{array}$ & $\begin{array}{c}0,374 * * * \\
{[0,135]}\end{array}$ & $\begin{array}{c}0,352 * * * \\
{[0,128]}\end{array}$ \\
\hline Betriebsteil ausgegliedert (Dummy) & $\begin{array}{c}0,027 \\
{[0,163]}\end{array}$ & $\begin{array}{c}0,221 \\
{[0,138]}\end{array}$ & $\begin{array}{c}0,197 \\
{[0,133]}\end{array}$ & $\begin{array}{c}0,221 \\
{[0,137]}\end{array}$ & $\begin{array}{c}0,196 \\
{[0,133]}\end{array}$ \\
\hline Betriebsteil eingegliedert (Dummy) & $\begin{array}{c}0,033 \\
{[0,180]}\end{array}$ & $\begin{array}{l}-0,009 \\
{[0,127]}\end{array}$ & $\begin{array}{c}0,011 \\
{[0,122]}\end{array}$ & $\begin{array}{l}-0,009 \\
{[0,127]}\end{array}$ & $\begin{array}{c}0,011 \\
{[0,122]}\end{array}$ \\
\hline Teilzeitanteil & $\begin{array}{l}0,186 \\
{[0,235]}\end{array}$ & $\begin{array}{l}0,303^{*} \\
{[0,155]}\end{array}$ & $\begin{array}{l}0,256 \\
{[0,162]}\end{array}$ & $\begin{array}{l}0,299 * \\
{[0,155]}\end{array}$ & $\begin{array}{c}0,254 \\
{[0,162]}\end{array}$ \\
\hline Frauenanteil & $\begin{array}{c}0,366 \\
{[0,288]}\end{array}$ & $\begin{array}{c}0,227 \\
{[0,141]}\end{array}$ & $\begin{array}{c}0,203 \\
{[0,153]}\end{array}$ & $\begin{array}{c}0,223 \\
{[0,141]}\end{array}$ & $\begin{array}{c}0,201 \\
{[0,153]}\end{array}$ \\
\hline Arbeiteranteil & $\begin{array}{c}0,498 \\
{[0,324]}\end{array}$ & $\begin{array}{c}0,038 \\
{[0,113]}\end{array}$ & $\begin{array}{c}0,032 \\
{[0,119]}\end{array}$ & $\begin{array}{c}0,036 \\
{[0,113]}\end{array}$ & $\begin{array}{c}0,030 \\
{[0,119]}\end{array}$ \\
\hline Qualifiziertenanteil & $\begin{array}{c}0,665 \\
{[0,284]}\end{array}$ & $\begin{array}{c}-0,237^{* *} \\
{[0,109]}\end{array}$ & $\begin{array}{c}-0,290 * * \\
{[0,115]}\end{array}$ & $\begin{array}{c}-0,244^{* *} \\
{[0,109]}\end{array}$ & $\begin{array}{c}-0,295^{* *} \\
{[0,115]}\end{array}$ \\
\hline Anzahl Beschäftigte (log) & $\begin{array}{l}3,778 \\
{[1,667]}\end{array}$ & $\begin{array}{c}0,701 * * * \\
{[0,078]}\end{array}$ & $\begin{array}{c}0,640 * * * \\
{[0,085]}\end{array}$ & $\begin{array}{c}0,694^{* * *} \\
{[0,079]}\end{array}$ & $\begin{array}{c}0,635^{* * *} \\
{[0,085]}\end{array}$ \\
\hline Anzahl Beschäftigte $\left(\log ^{2}\right)$ & $\begin{array}{c}17,085 \\
{[13,773]}\end{array}$ & $\begin{array}{c}0,002 \\
{[0,008]}\end{array}$ & $\begin{array}{c}0,006 \\
{[0,009]}\end{array}$ & $\begin{array}{c}0,002 \\
{[0,008]}\end{array}$ & $\begin{array}{c}0,007 \\
{[0,009]}\end{array}$ \\
\hline Branche (Dummies) & & Ja & Ja & Ja & Ja \\
\hline Jahr (Dummies) & & Ja & Ja & Ja & Ja \\
\hline Konstante & & $\begin{array}{c}-2,829 * * * \\
{[0,280]}\end{array}$ & $\begin{array}{c}-2,696 * * * \\
{[0,303]}\end{array}$ & $\begin{array}{c}-2,842 * * * \\
{[0,281]}\end{array}$ & $\begin{array}{c}-2,703 * * * \\
{[0,303]}\end{array}$ \\
\hline Anzahl Firmen & 1412 & 1412 & 1412 & 1412 & 1412 \\
\hline Anzahl Beobachtungen & 3679 & 3679 & 3679 & 3679 & 3679 \\
\hline linkszensierte Beobachtungen & 2368 & 2368 & 2368 & 2368 & 2368 \\
\hline unzensierte Beobachtungen & 1311 & 1311 & 1311 & 1311 & 1311 \\
\hline Pseudo R² (McFadden) & & 0,212 & & 0,212 & \\
\hline $\begin{array}{l}\text { Standardabweichung bzw. Standardfehler in } \\
* * 5 \%-, * * * 1 \%-\text { Niveau. } \\
\text { Quelle: Hannoveraner Firmenpanel } 2000-20\end{array}$ & nmern. Signifi & $\begin{array}{l}\text { t auf dem * } 10 \\
\text { es Autors. }\end{array}$ & & & $\begin{array}{l}\text { Hans Böckler } \\
\text { Stiftung }\end{array}$ \\
\hline
\end{tabular}

über den gesamten Analysezeitraum 3.679 Beobachtungen von 1.412 Betrieben. Etwa $36 \%$ haben berichtet, dass Arbeitnehmer im ersten Halbjahr des Jahres freiwillig gekündigt haben. Die Spalten drei und vier beinhalten die Koeffizienten und Standardfehler für die erste Spezifikation. Die Existenz eines Betriebsrates verringert sowohl im gepoolten als auch im RandomEffects Tobit Modell die logarithmierte Anzahl der freiwilligen Kündigungen in einem Betrieb mit einer statistischen Sicherheit von $1 \%$, wobei der Einfluss unter Berücksichtigung von unbeobachtbarer Heterogenität etwas geringer ist. Auch in tarifgebundenen Betrieben ist die Anzahl der arbeitnehmerseitigen Kündigungen geringer. Jedoch sind die Koeffizienten signifikant kleiner als in Betrieben mit Betriebsräten und auch nur im gepoolten Modell signifi- teils ist mit einer signifikant höheren Anzahl von arbeitnehmerseitigen Personalabgängen korreliert, und auch der Koeffizient einer Ausgliederung ist positiv. Ebenso haben Betriebe mit einem höheren Anteil von Teilzeitbeschäftigten, Frauen und Arbeitern tendenziell mehr Abgänge. Dagegen ist der Anteil von qualifizierten Arbeitskräften signifikant negativ mit freiwilligen Kündigungen von Arbeitnehmern korreliert. Dieser Befund könnte darauf hindeuten, dass Personalabgänge und die daraus folgenden Neueinstellungen von qualifizierten Arbeitnehmern mit höheren Kosten verbunden sind und der Betrieb daher einen stärkeren Anreiz hat, auf Meinungsäußerungen von Qualifizierten zu hören, um deren Abgänge zu vermeiden.

In Tabelle 3 wurden die Koeffizienten der Variablen aus den vorherigen Schätzungen in den prozentualen Einfluss auf die Anzahl der freiwilligen Kündigungen von Arbeitnehmern umgerechnet, um die quantitative Interpretation zu vereinfachen. Betriebe mit einem Betriebsrat haben etwa $25 \%$ und tarifgebundene Betriebe etwa $10 \%$ weniger arbeitnehmerseitige Kündigungen als Betriebe ohne die jeweilige Institution. In der differenzierten Betrachtung hat die alleinige Existenz eines Betriebsrates immer noch einen Einfluss von ungefähr $20 \%$, und einer alleinigen Tarifbindung kommt ein Einfluss von ungefähr $7 \%$ zu. Es zeigt sich somit, dass zwar sowohl die Existenz eines Betriebsrates als auch eine Tarifbindung zu weniger freiwilligen Kündigungen durch die Arbeitnehmer führen, aber der Einfluss des Betriebsrates ist deutlich stärker, was auf seine Voice-Funktion zurückgeführt werden kann (Hypothesen 1 und 2). Den stärksten Einfluss auf die Vermeidung freiwilliger Kündigungen hat jedoch die Koexistenz von Betriebsrat und Tarifbindung, die mit einer Reduzierung von über $30 \%$ einhergeht. Dies entspricht der theoretischen Überlegung, dass Betriebsräte effektiver in der Vermeidung arbeitnehmerseitiger Kündigungen sind, falls durch eine Tarifbindung Rahmenbedingungen exogen vorgegeben sind, sodass Verteilungskonflikte weniger stark in den Betrieben ausgefochten werden müssen. Möglicherweise erfolgt in tarifgebundenen Betrieben auch eine stärkere beratende und personelle Unterstützung der Betriebsräte durch die Gewerkschaften (Hypothese 3).

Neben den Schätzungen für alle Betriebe wurden Schätzungen für die Betriebs- 
größenklassen 21 bis 100 und 101 bis 500 Beschäftigte sowie für das Verarbeitende Gewerbe und den Dienstleistungssektor durchgeführt, deren Ergebnisse ebenfalls in Tabelle 3 zusammengefasst sind. Insgesamt wurden die Ergebnisse für alle Betriebe bestätigt. Der Einfluss von Betriebsräten ist größer als der einer Tarifbindung. Den stärksten Einfluss in allen Stichproben hat die Koexistenz von Betriebsrat und Tarifbindung. Es gibt jedoch auch Unterschiede in den Schätzungen. So hat eine Tarifbindung in größeren Betrieben teilweise sogar einen positiven Einfluss auf die Anzahl der freiwilligen Kündigungen, wobei eine Koexistenz mit einem Betriebsrat weiterhin einen starken negativen Einfluss ausübt. Ferner scheint der Betriebsratseffekt in der Dienstleistungsbranche stärker zu sein als im Verarbeitenden Gewerbe. Da die Verbreitung von Interessenvertretungen in der Dienstleistungsbranche deutlich geringer ist, könnte dieser Befund darauf hindeuten, dass Arbeitnehmer die Interessenvertretungen schätzen und nicht freiwillig kündigen, um das Risiko zu umgehen, in einem interessenvertretungsfreien Betrieb eine neue Beschäftigung zu finden.

\section{Fazit}

Der Grundgedanke der Analyse in diesem Beitrag ist, dass Arbeitnehmer freiwillig kündigen, falls ihr Nutzen aus einer Alternative größer ist als ihr Nutzen aus ihrer aktuellen Arbeit. Arbeitnehmer können beispielsweise in eine andere Firma mit besseren Arbeitsbedingungen wechseln oder eine vorübergehende oder dauerhafte Arbeitslosigkeit wählen. Sowohl Betriebsräte als auch Tarifverträge können zu einer allgemeinen Verbesserung der Arbeitsbedingungen und somit zu einem höheren Nutzen aus Arbeit beitragen, wodurch es selte-

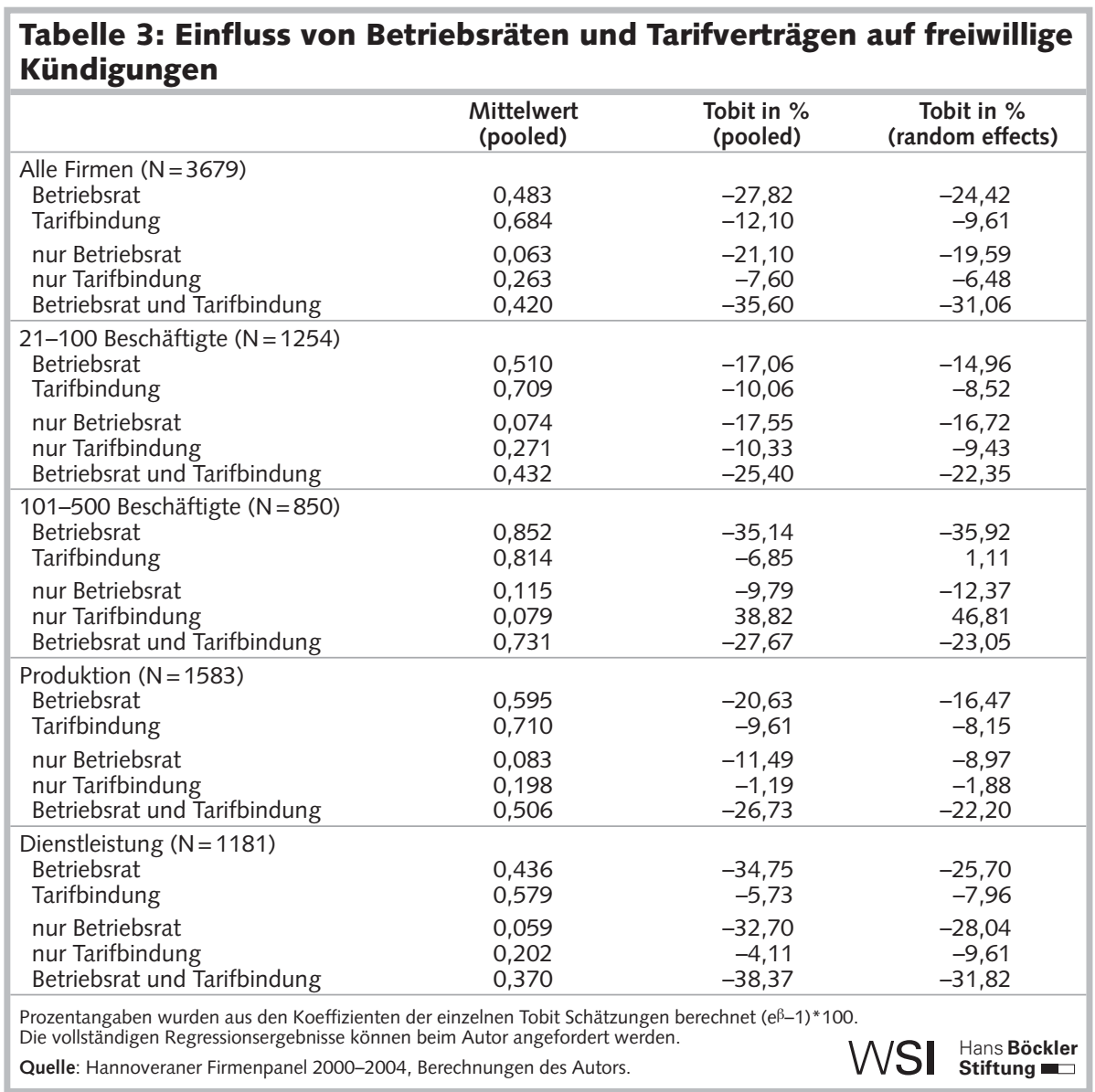

ner zu freiwilligen Kündigungen kommt. Ferner haben Betriebsräte eine VoiceFunktion, da kündigungswillige Arbeitnehmer ihre Unzufriedenheit mit bestimmten Arbeitsbedingungen gegenüber ihrer betrieblichen Interessenvertretung äußern können, die dann unterstützend tätig wird. Es zeigt sich zwar, dass der Einfluss von Betriebsräten stärker ist als der Einfluss einer Tarifbindung, aber am wirkungsvollsten ist die Koexistenz beider Institutionen für die Reduzierung arbeitnehmerseitiger Personalabgänge. Denn Betriebsräte sind effektiver in ihrer Arbeit, wenn Verteilungskonflikte großteils außerhalb des Betriebes in Tarifverhandlungen ausgetragen werden.
Die Ergebnisse zeigen, dass es sowohl für Arbeitnehmer als auch für Arbeitgeber positive Effekte von Betriebsräten und Tarifverträgen gibt. Denn beide Seiten haben im Allgemeinen ein Interesse an der Vermeidung von Eigenkündigungen durch Arbeitnehmer. Die Arbeitnehmerseite erspart sich den Wechsel in eine Alternative, der auch mit Mobilitätskosten verbunden ist. Die Arbeitgeberseite spart Kosten der Neueinstellung und Einarbeitung und hat zudem eher Anreize, in betriebsspezifisches Humankapital zu investieren, das für die betriebliche Produktivität wichtig sein kann. 
Addison, J. T./Belfield, C. R. (2004): Union voice, in: Journal of Labor Research 25, pp. 563-597

Addison, J. T./Bellmann, L./Schnabel, C./Wagner, J. (2004): The reform of the German Works Constitution Act: A critical assessment, in: Industrial Relations 43, pp. 392-420

Addison, J. T./Schnabel, C./Wagner, J. (2004): The course of research into the economic consequences of German works councils, in: British Journal of Industrial Relations 42, pp. 255-281

Addison, J. T./Gerlach, K. (1983): Gewerkschaften und Produktivität: Fehlallokation von Ressourcen oder Produktivitätssteigerung?, in: Zeitschrift für die gesamte Staatswissenschaft 139, S. 215-228

Dilger, A. (2002): Ökonomik betrieblicher Mitbestimmung: Die wirtschaftlichen Folgen von Betriebsräten, München/Mering

Freeman, R. B. (1976): Individual mobility and union voice in the labor market, in: American Economic Review 66, S. 361-368

Freeman, R. B. (1980): The exit-voice tradeoff in the labor market: unionism, job tenure, quits, and separations, in: Quarterly Journal of Economics 94, pp. 643-673

Freeman, R. B./Lazear, E. P. (1995): An economic analysis of works councils, in: Rogers, J./Streeck, W. (eds.): Works councils: consultation, representation and cooperation in industrial relations, Chicago, pp. 27-50

Freeman, R. B./Medoff, J. L. (1984): What do unions do? New York Frick, B. (1996): Co-determination and personnel turnover: the German experience, in: Labour 10, pp. 407-430

Frick, B. (2006): Codetermination and personnel turnover: ten years later, in: Schmollers Jahrbuch 126(2), pp. 307-312

Frick, B./Möller, I. (2003): Mandated works councils and firm performance: labour productivity and personnel turnover in German establishments, in: Schmollers Jahrbuch 123, pp. 423-454
Gerlach, K./Hübler, O./Meyer, W. (2003): The Hannover Firm Panel (HFP), in: Schmollers Jahrbuch 123, S. 463-470

Hirschman, A. O. (1970): Exit, voice and loyality, Cambridge/Mass. Hübler, O./Jirjahn, U. (2003): Works councils and collective bargaining in Germany: the impact on productivity and wages, in: Scottish Journal of Political Economy 50, pp. 471-491

Jirjahn, U. (2005): Ökonomische Wirkungen des novellierten Betriebsverfassungsgesetzes - Was können wir vor dem Hintergrund zunehmender Globalisierung und veränderter arbeitsorganisatorischer Bedingungen erwarten?, in: Zeitschrift für Arbeitsmarktforschung 38, S. 241-267 Jirjahn, U. (2006): Ökonomische Wirkungen der Mitbestimmung in Deutschland. Überblick über den Stand der Forschung und Perspektiven für zukünftige Studien, in: Sozialer Fortschritt 55, S. 215-226

Kraft, K. (1986): Exit and voice in the labour market: an empirical study of quits, in: Zeitschrift für die gesamte Staatswissenschaft 142, S. 697715

Kraft, K. (2006): On estimating the effect of co-determination on personnel turnover, in: Schmollers Jahrbuch 126, S. 287-305

Miller, P./Mulvey, C. (1991): Australian evidence on the exit/voice model of the labor market, in: Industrial and Labor Relations Review 45, pp. 44-57

Müller-Jentsch, W. (1995): Germany: From collective voice to comanagement, in: Rogers, J./Streeck, W. (eds.): Works councils: consultation, representation and cooperation in industrial relations, Chicago, pp. 53-78

TNS Infratest (2005): IAB Betriebspanel Arbeitgeberbefragung 2004, München 